\title{
Identification of highly conserved domains in hemagglutinin associated with the receptor binding specificity of influenza viruses: 2009 H1N1, avian H5N1, and swine H1N2
}

\section{Wei Hu}

Department of Computer Science, Houghton College, Houghton, New York, USA.

Email:wei.hu@houghton.edu

Received 11 December 2009; revised 14 December 2009; accepted 15 December 2009.

\begin{abstract}
The hemagglutinin (HA) of influenza viruses facilitates receptor binding and membrane fusion, which is the initial step of virus infection. Human influenza viruses preferentially bind to receptors with $\alpha 2-6$ linkages to galactose (SAd2,6Gal), whereas avian influenza viruses prefer receptors with $\alpha 2-3$ linkages to galactose (SAd2,3Gal). The current 2009 H1N1 pandemic is caused by a novel influenza $A$ virus that has its genetic materials from birds, humans, and pigs. Its pandemic nature is characterized clearly by its dual binding to the $\alpha 2-3$ as well as $\alpha 2-6$ receptors, because the seasonal human H1N1 virus only binds to the $\alpha 2-6$ receptor. In a previous study, the informational spectrum method (ISM), a bioinformatics technique, was applied to uncover one highly conserved region in the HA protein associated with receptor binding preference in each of various influenza subtypes. In the present study, we extended the previous work by discovering multiple such domains in HA of 2009 H1N1 and avian H5N1 to expand our repertoire of known key regions in $\mathrm{HA}$ responsible for receptor binding affinity. Three such domains in HA of 2009 H1N1 were found at residue positions 106 to 130,150 to 174 , and 191 to 221 , and another three domains in HA of avian H5N1 were located at residue positions 46 to 65,136 to 153 , and 269 to 286 . These identified domains could be utilized as therapeutic and diagnostic targets for the prevention and treatment of influenza infection.
\end{abstract}

Keywords: Binding Specificity; Discrete Fourier Transform; Electron-Ion Interaction Potential; Entropy; Hemagglutinin; Influenza; Informational Spectrum Method

\section{INTRODUCTION}

Influenza A viruses have two surface proteins, hemag- glutinin (HA) and neuraminidase (NA). HA is a homotrimer, in which each monomer comprises two subdomains, HA1 and HA2. HA1 initializes the contact with the cell membrane and HA2 mediates membrane fusion. The first step in the infection of influenza viruses is binding of their surface protein HA to sialylated glycan receptors on the host cells. In general, human influenza and avian viruses preferentially bind to the $\alpha 2-3$ sialylated and $\alpha 2-6$ sialylated glycan receptors, respectively. Pigs have receptors for both human and avian influenza viruses on their tracheal epithelial cells, thus they can serve as a mixing vessel to re-assort genes from different species to make new influenza viruses.

The interaction between HA and its receptors has been studied biologically, genetically, and structurally. The different binding phenotypes of human and avian influenza viruses suggest that the avian viruses could not readily infect humans. However, the human infection by H5N1 chicken viruses in Hong Kong in 1997 implied for the first time that it is possible for avian viruses to infect humans directly, which was explained in part by the finding that human airway epithelium harbors $\alpha 2-3$ linked sialic acids on ciliated cells [1]. It is believed that HA binding affinity for receptors is a critical factor of host switch. In addition to the current 2009 pandemic H1N1, the past three flu pandemics, the Spanish flu in 1918, the Asian flu in 1957, and the Hong Kong flu in 1968, all had arisen through reassortment among avian, human, and swine strains. Hence, the importance of expanding our knowledge on the receptor-binding affinity of the influenza viruses is apparent.

Various approaches such as structural analysis, protein evolution, and mathematical modeling have been used to study the interactions between HA and its receptors. To quantitatively elucidate the binding specificity of HA to avian and human receptors, the interaction energy between HA and its receptors were analyzed with the ab initio fragment molecular orbital (FMO) method [2], which clarified the role of the mutated residues Glu190 and Gln226 in the binding patterns of H5 HA. The report 
in [3] discovered that the mutations at positions 182 and 192 in HA1 independently switch H5N1 virus binding preferences from avian to human type, which could serve as molecular markers for measuring the pandemic potential of $\mathrm{H} 5 \mathrm{~N} 1$ isolates.

Using sequence analysis and homology modeling [4], the HA protein of $2009 \mathrm{H} 1 \mathrm{~N} 1$ was found to have the signature amino acid Asp190 and Asp225 known to confer binding affinity to $\alpha 2-6$ sialylated glycan receptors. The mutation Glu190Asp between avian and human H1 HA normally would lead to the loss of a critical contact with a2-3 glycans, which, however, was compensated by the presence of Lys145 in HA of 2009 H1N1. There were four loops in 2009 H1N1 HA, 130 loop, 140 loop, 150 loop, and 220 loop, each containing one Lys, to form a positively charged 'lysine fence' at the base of the binding site to support optimal contacts with the $\alpha 2-6$ and $\alpha 2-3$ receptors. Based on this structural analysis, it was predicted that the HA protein of $2009 \mathrm{H} 1 \mathrm{~N} 1$ virus can bind to the $\alpha 2-6$ as well as $\alpha 2-3$ sialylated glycan receptors, which was verified later by the carbohydrate microarray analysis in [5].

There were several other efforts in expanding the knowledge on 2009 H1N1. One study [6] investigated the three aspects of NA: the mutations and co-mutations, the stalk motifs, and the phylogenetic analysis. The potential mutations and strongly co-mutated positions of NA were found. A special NA stalk motif of high virulence, which was dominant in the past H5N1 strains, was discovered in H1N1 in 2009 for the first time. Another study [7] focused on HA and the interaction between HA and NA. The mutations of HA in $2009 \mathrm{H} 1 \mathrm{~N} 1$ were found and mapped to the 3D homology model of $\mathrm{H} 1$, and the mutations on the five epitope regions on H1 were identified. The distinct response patterns of HA to the changes of NA stalk motifs were discovered, illustrating the functional dependence between HA and NA. With help from the results of the first study in [6], two co-mutation networks were uncovered, one in HA and one in NA, where each mutation in one network co-mutates with the mutations in the other network across the two proteins HA and NA. These two networks residing in HA and NA separately may provide a functional linkage between the mutations that can change the drug binding sites in NA and those that can affect the host immune response or vaccine efficacy in HA.

The informational spectrum method (ISM) [8] is a bioinformatics technique to study the biological functions of proteins with their physicochemical properties, which first translates a protein sequence into a numerical sequence based on each amino acid's electron-ion interaction potential (EIIP) and then the discrete Fourier transformation (DFT) is applied to it to create an informational spectrum. It is believed that the protein functions including the protein-protein interaction are encoded in the peaks of the informational spectrum.

Highly conserved regions in a protein sequence usually have functional or structural values. The active site of enzymes and the binding sites of protein receptors are typical examples of these highly conserved regions.

In references [9-11], the ISM was applied successfully to characterize the conserved information pertinent to the interaction between HIV-1 and their CD4, CCR5, and CXCR4 receptors. In [12,13] the same research group applied the ISM to investigate the interaction between HA and its receptors. Their findings showed that HA1 of different flu subtypes encodes one highly conserved domain that might be determinants of HA binding affinity. Our goal in this study is to extend the results in $[12,13]$ by identifying multiple domains in HA1 associated with each receptor interaction pattern. These conserved domains in HA1 might be used to develop targets for new drugs and infection control.

In $[12,13]$ it was found that the consensus informational spectrum (CIS) of HA1 of influenza strains have the following characteristic dominant peaks at different IS frequencies as presented in Table 1. In this study, $\mathrm{F}(0.295)$ will be referred to as pandemic human H1N1 receptor interaction frequency, $\mathrm{F}(0.055)$ as swine receptor interaction frequency, $\mathrm{F}(0.076)$ as avian receptor interaction frequency, and $\mathrm{F}(0.236)$ as seasonal human H1N1 receptor interaction frequency.

Table 1. Characteristic IS frequencies of HA proteins in 2009 H1N1, swine H1N1/H1N2, avian H5N1, and seasonal human H1N1.

\begin{tabular}{ccccc}
\hline Subtype & 2009 H1N1 & Swine H1N2/H1N1 & Avian H5N1 & Seasonal human H1N1 \\
\hline Frequency & $\mathrm{F}(0.295)$ & $\mathrm{F}(0.055)$ & $\mathrm{F}(0.076)$ & $\mathrm{F}(0.236)$ \\
\hline
\end{tabular}

Table 2. The receptor recognition domains of HA proteins in H1N1, H3N2, H5N1, and H7N7 influenza viruses.

\begin{tabular}{lcc}
\hline \multicolumn{1}{c}{ Strain } & Frequency & Residues \\
\hline A/California/04/2009 (H1N1) & $\mathrm{F}(0.295)$ & $284-326$ \\
A/Hong Kong/213/03 (H5N1) & $\mathrm{F}(0.076)$ & $42-75$ \\
A/New Caledonia/20/99 (H1N1) & $\mathrm{F}(0.236)$ & $262-295$ \\
A/New York/383/2004 (H3N2) & $\mathrm{F}(0.363)$ & $57-90$ \\
A/equine/Prague/56 (H7N7) & $\mathrm{F}(0.285)$ & $28-61$ \\
A/Egypt/0636-NAMRU3/2007(H5N1) & $\mathrm{F}(0.236)$ & $99-132$ \\
A/South Carolina/1/18 (H1N1)) & $\mathrm{F}(0.258)$ & $87-120$ \\
\hline
\end{tabular}




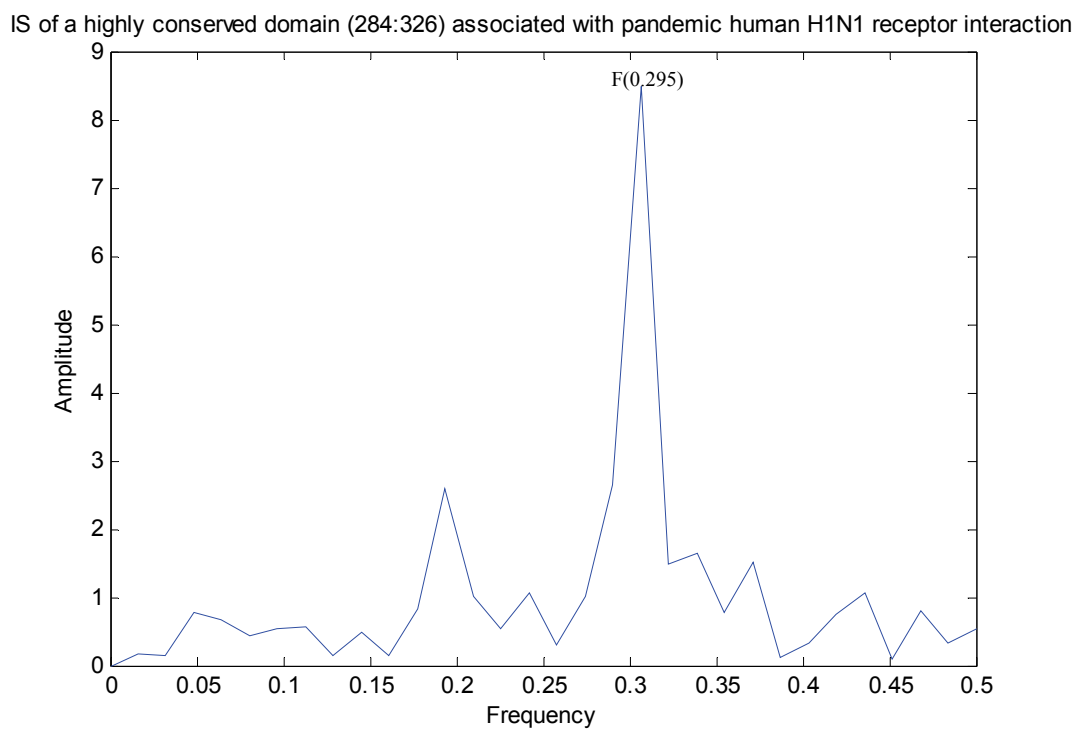

Figure 1. The informational spectrum of a highly conserved domain in 2009 H1N1 found in [12], which is also a part of Figure 5 in [12].

Their analysis also found the following receptor recognition domains in HA proteins from H1N1, H3N2, H5N1, and H7N7 (Table 2). The IS of one such domain in A/California/04/2009 was displayed in Figure 1, which was a reproduced figure in [12].

\section{MATERIALS AND METHODS}

\subsection{Sequence Data}

All HA sequences were retrieved from the Influenza Virus Resource (http://www.ncbi/nlm.nih.giv/genomes/FLU/ FLU.html) of the National Center for Biotechnology Information (NCBI) on Nov. 20, 2009. Only the full length and unique sequences were selected. There were 450 HA sequences of human 2009 H1N1, 1228 HA sequences of avian H5N1 from 1959 to 2009, and $83 \mathrm{HA}$ sequences of swine H1N2 from 1980 to 2009. All the sequences used in the study were aligned with MAFFT [14].

\subsection{Entropy}

In information theory [15], entropy is a measure of disorder or randomness associated with a random variable. Let $x$ be a discrete random variable that has a set of possible values $\left\{a_{1}, a_{2}, a_{3}, \ldots a_{n}\right\} \quad$ with probabilities $\left\{p_{1}, p_{2}, p_{3}, \ldots p_{n}\right\}$ where $P\left(x=a_{i}\right)=p_{i}$. The entropy $\mathrm{H}$ of $x$ is

$$
H(x)=-\sum_{i} p_{i} \log p_{i}
$$

In the current study, each of the n columns in a multiple sequence alignment of a set of HA sequences of $\mathrm{N}$ residues is considered as a discrete random variable $x_{i}$
$(1 \leq \mathrm{i} \leq \mathrm{N})$ that takes on one of the $20(\mathrm{n}=20)$ amino acid types with some probability. $H\left(x_{i}\right)$ has its minimum value 0 if all the residues at position $i$ are the same, and achieves its maximum if all the 20 amino acid types appear with equal probability at position $\mathrm{i}$, which can be verified by the Lagrange multiplier technique. A position of high entropy means that the amino acids are often varied at this position. $H\left(x_{i}\right)$ measures the genetic diversity at position i in our current study. A brief overview of the extensive applications of entropy in sequence analysis, in particular the flu virus sequences, can be found in [6].

\subsection{Important Sites in HA}

Although there is a great variation due to high selection pressure in the HA1 sequences of various flu subtypes, the active site of HA1 is well conserved, which is located in a cleft composed of the residues $91,150,152$, $180,187,191$, and 192 [16]. The three amino acids at positions 187, 191 and 192 are a part of the 190 helix. The active site cleft of HA is formed by its right edge (131_GVTAA) and left edge (221_RGQAGR) (H1 numbering), which are also commonly referred to as the 130 loop and 220 loop, respectively [17].

The human immune system responds primarily to the five epitope regions, $\mathrm{A}, \mathrm{B}, \mathrm{C}, \mathrm{D}$, and $\mathrm{E}$, of the HA protein in H1N1. Table 3 presents the 160 amino acids on the five epitope regions of HA in H1 subtype as discovered in [18].

\subsection{Informational Spectrum Method}

The informational spectrum method is a bioinformatics 
technique that can be utilized to analyze protein sequences. Prior to this analysis, the protein sequences have to be translated into numerical sequences. One such approach is to assign each amino acid to its electron-ion interaction potential (EIIP), which represents the average energy of the valence electrons in the amino acid (Table 4). The application of EIIP to protein function analysis assumes that the strength of the electromagnetic field surrounding the protein is indicative of its biological function. This method was successful in revealing various protein properties [8].

The numerical sequence $x(m)$ of a protein sequence is transformed into the frequency domain using DFT. The DFT coefficients $x(n)$ are defined as

$$
X(n)=\sum x(m) e^{-j\left(\frac{2 \pi}{N}\right) n m} \quad n=1,2, \ldots N / 2
$$

where $\mathrm{N}$ is the length of sequence $x(m)$.

The energy density spectrum is defined as

$$
S(n)=X(n) X^{*}(n)=|X(n)|^{2}, \quad n=1,2, \ldots N / 2
$$

The informational spectrum (IS) of a sequence $x(m)$ comprises the frequencies and the amplitudes of its DFT.

Peak frequencies of IS of a protein sequence reflect its biological or biochemical functions. To determine the same biological or biochemical functions of a group of protein sequences, a consensus informational spectrum (CIS) can be used, which is defined as the product of energy density spectrum $S(n)$ of each sequence in the group. A measure of similarity for each peak is a signal-to-noise ratio $(\mathrm{S} / \mathrm{N})$, which is defined as a ratio of signal density to the mean value of the whole spectrum [12]. The theory of CIS [8] states that:

1) One peak only exits for a group of protein sequences sharing the same biological function.

2) No signal peak exists for biologically unrelated protein sequences.

3) Peak frequencies are different for different biological functions.

\begin{tabular}{|c|c|c|}
\hline Epitope & Amino acids & Number of amino acids \\
\hline A & $\begin{array}{l}118,120,121,122,126,127,128,129,132,133,134,135,137,139,140,141,142,143,146,14 \\
7,149,165,252,253\end{array}$ & 24 \\
\hline $\mathrm{B}$ & $\begin{array}{l}\text { 124,125,152,153,154,155,156,157,160,162,183,184,185,186,187,189,190,191,193,19 } \\
4,195,196\end{array}$ & 22 \\
\hline $\mathrm{C}$ & $\begin{array}{l}34,35,36,37,38,40,41,43,44,45,269,270,271,272,273,274,276,277,278,283,288,292,2 \\
95,297,298,302,303,305,306,307,308,309,310\end{array}$ & 32 \\
\hline $\mathrm{D}$ & $\begin{array}{l}170,171,172,173,174,176,179,198,200,202,204,205,206,207,208,209,210,211,212,21 \\
3,214,215,216,222,223,224,225,226,227,235,237,239,241,243,244,245\end{array}$ & 48 \\
\hline E & $\begin{array}{l}47,48,50,51,53,54,56,57,58,66,68,69,70,71,72,73,74,75,78,79,80,82,83,84,85,86,102, \\
257,258,259,260,261,263,267\end{array}$ & 34 \\
\hline
\end{tabular}

Table 3. Amino acids on epitopes A, B, C, D, and E of H1 subtype (A/California/04/2009 numbering) from [18].

Table 4. The electron-ion interaction potential (EIIP) of amino acids used to encode amino acids.

\begin{tabular}{cccc}
\hline Amino acid & EIIP & Amino acid & EIIP \\
\hline L & 0.0000 & Y & 0.0516 \\
I & 0.0000 & W & 0.0548 \\
N & 0.0036 & Q & 0.0761 \\
G & 0.0050 & M & 0.0823 \\
E & 0.0057 & S & 0.0829 \\
V & 0.0058 & C & 0.0829 \\
P & 0.0198 & T & 0.0941 \\
H & 0.0242 & F & 0.0946 \\
K & 0.0371 & R & 0.0959 \\
A & 0.0373 & D & 0.1263 \\
\hline
\end{tabular}




\subsection{Three Consensus HA1 Sequences of 2009 Human H1N1, Avian H5N1, and Swine N1N2}

We employed MAFFT to align the three consensus HA1 sequences of $2009 \mathrm{H} 1 \mathrm{~N} 1$, avian H5N1, and swine H1N2 (Figure 2). Each consensus sequence was then used in the ISM analysis to find the highly conserved domains in HA1 of different influenza subtypes.

\section{RESULTS}

As demonstrated in $[12,13]$, the HA1 sequences in various influenza subtypes had a distinct propensity to interact with a specific receptor, and there was a region in HA1 encoding highly conserved information that might be associated with the binding preference. The main task of the present study is to explore the other parts of the HA1 sequences to find domains of the same biological function. Entropy of HA1 in each subtype of 2009 H1N1, swine H1N2, and avian H5N1 was calculated, which illustrated the most conserved positions in the HA1 sequences of each subtype (Figures 5, 7, and 10). The ISM bioinformatics technique was applied to the three consensus HA1 sequences as presented in Figure 2 to uncover the conserved domains in HA1, which might modulate receptor specificity in each subtype. In contrast, the ISM analysis in $[12,13]$ was applied to a particular selected strain in a subtype such as A/California/04/2009 in $2009 \mathrm{H} 1 \mathrm{~N} 1$ to find a conserved region. On the whole, the conserved domains discovered by our approach using a consensus sequence had more coverage to different sequences in a subtype than the single strain approach in $[12,13]$.

\subsection{Conserved Domains in HA1 of 2009 H1N1}

We discovered three domains in HA1 of 2009 H1N1, which were located at residue positions 106 to 130,150 to 174 , and 191 to 221 . The consensus sequences of theses domains were SSVSSFERFEIFPKTSSWPNHDSNK, $\begin{array}{lll}1 & 2009 & \text { H1N1 } \\ 2 & \text { Swine H1N1 } \\ 3 & \text { Avian H5N1 }\end{array}$

12009 H1N1

2 Swine H1N2

3 Avian $\mathrm{H} 5 \mathrm{~N} 1$

12009 H1N1

2 Swine H1N2

3 Avian $\mathrm{H} 5 \mathrm{~N} 1$

12009 H1N1

2 Swine H1N2

3 Avian $\mathrm{H} 5 \mathrm{~N} 1$

DTLCIGYHANNSTDTVDTVLEKNVTVTHSVNLLEDKHNGKLCKLRGVAPLHLGKCNIAGW DTLCIGYHANNSTDTVDTVLEKNVTVTHSVNLLEDRHNGKLCKLRGVAPLHLGKCNIAGW DQICIGYHANNSTEQVDT IMEKNVTVTHAQDILEKTHNGKLCDLDGVKPLILRDCSVAGW

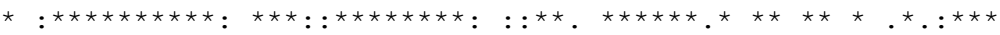

ILGNPECESLSTASSWSYIVETSSSDNGTCYPGDFIDYEELREQLSSVSSFERFEIFPKT LLGNPECESLFTASSWSYIVETSNSDNGTCYPGDFINYEELREQLSSVSSFERFEIFPKE LLGNPMCDEFINVPEWSYIVEKANPANDLCYPGNFNDYEELKHLLSRINHFEKIQIIPK-

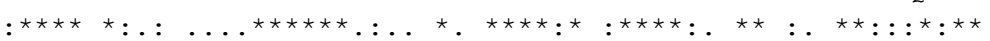

121 right edge 180 SSWPNHDSNKGVTAACPHAGAKS FYKNLIWLVKKGNSY PKLSKSY INDKGKEVLVLWGIH SSWPNHDTNRGVTAACPHAGANSFYRNLIWLVKKGNSYPKLSKSY INNKEKEVLVLWGIH SSWSDHEASSGVSSACPYQGRSSFFRNVVWLIKKNNAYPTI KRSYNNTNQEDLLVLWGIH

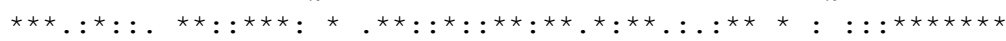

$181 \quad 240$ HPSTSADQQSLYQNADAYVFVGSSRYSKKFKPEIAIRPKVRDQEGRMNYYWTLVEPGDKI HPSTSADQQSLYQNADAYVFVGSSHYSKKFTPEIAKRPKVRDQAGRMNYYWTLVEPGDTI HPNDAAEQTRLYQNPTTYISVGTSTLNQRLVPKIATRSKVNGQSGRMEFFWTILKPNDAI

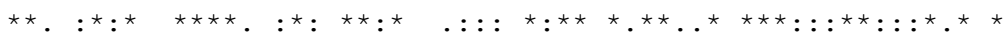

241

TFEATGNLVVPRYAFAMERNAGSGI I ISDTPVHDCNTTCQTPKGAINTSLPFQNIHPITI TFEATGNLVVPRYAFALKRGSGSGI I ISDTSVHDCNTTCQTPKGAINTSLPFQNIHPVTI NFESNGNF IAPEYAYKIVKKGDST IMKSELEYGNCNTKCQTPMGA INSSMPFHNIHPLTI

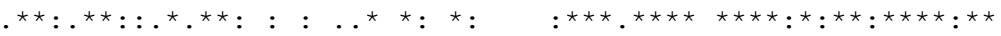

Swine H1N2

3 Avian $\mathrm{H} 5 \mathrm{~N} 1$

12009 H1N1

2 Swine H1N2

3 Avian $\mathrm{H} 5 \mathrm{~N} 1$

301328

GKCPKYVKSTKLRLATGLRNVPSIQSRGECPKYVKSTKLRMATGLRNIPSIQSRGECPKYVKSNRLVLATGLRNSPQRERRR

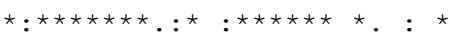

Figure 2. Multiple sequence alignment of three consensus HA1 sequences in 2009 H1N1, swine H1N2, and avian H5N1. The binding sites in HA are colored in red, and the left and right edges of the binding cleft are printed in boldface type. 

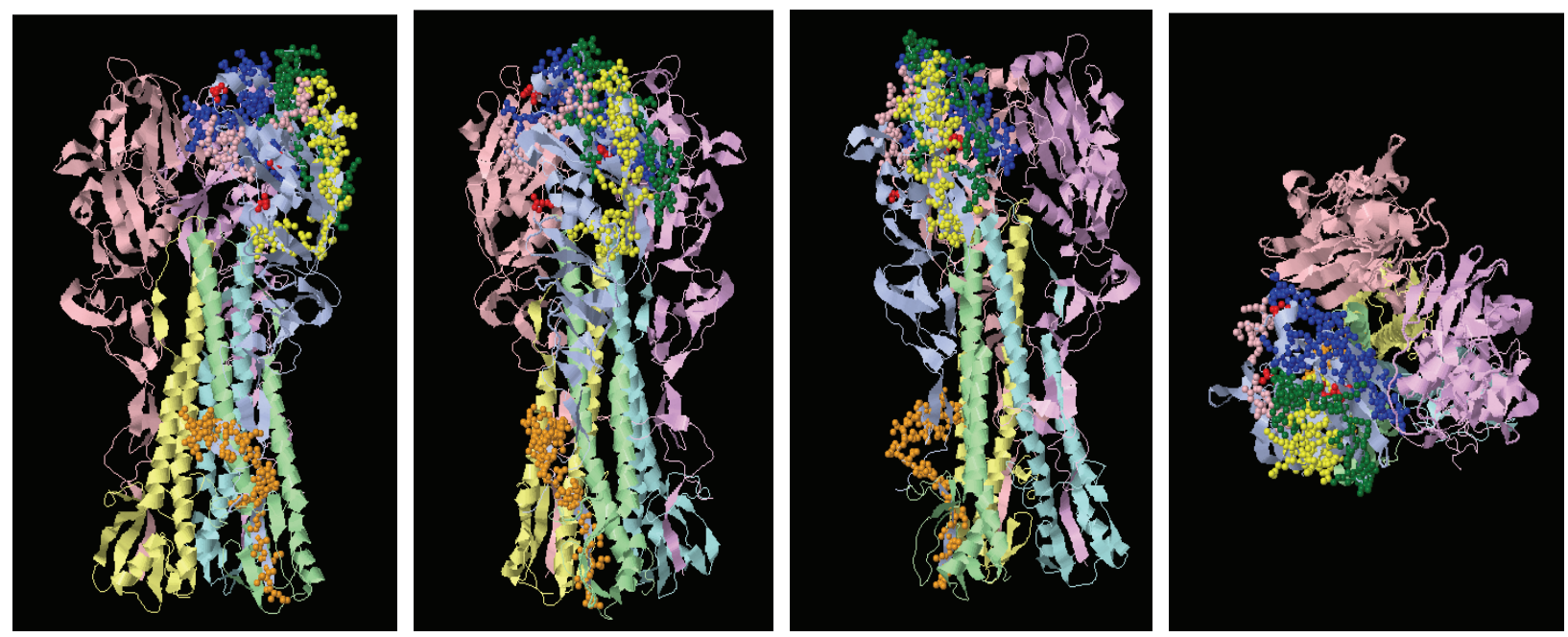

Figure 3. The four plots show in 3D structure the four highly conserved domains found in this study, three domains in 2009 H1N1 and one domain in swine H1N2. Binding sites are colored in red, two edges of the binding cleft in pink, domain 106:130 in 2009 H1N1 in yellow, domain 150:174 in 2009 H1N1 in green, domain 191:221 in 2009 H1N1 in blue, and domain 1:29 in swine H1N2 in orange (PDB code: 1RU7).
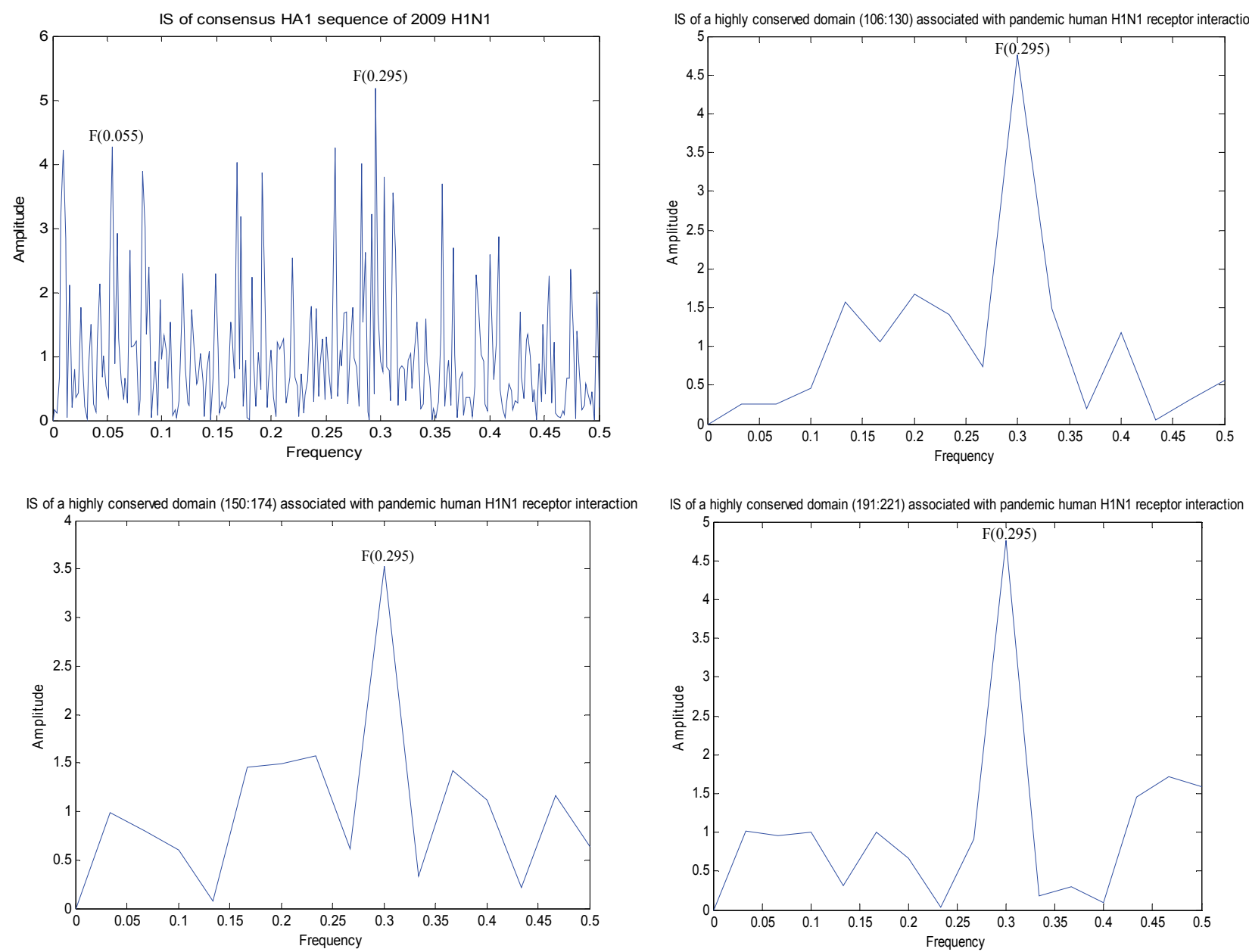

Figure 4. The top left plot shows the informational spectrum of consensus HA1 sequence in 2009 H1N1. The other three plots show the informational spectrum of the three conserved domains in 2009 H1N1 found in this study, 106:130, 150:174, and 191:221, respectively. 
WLVKKGNSYPKLSKSYINDKGKEVLV, and LYQNDAYVFVGSSRYSKKFKPEIAIRPKVR, respectively, and the ISs of these three domains and the consensus HA1 sequence in 2009 H1N1 were plotted in Figure 4. The IS of the consensus HA1 sequence in 2009 H1N1displayed a dominant peak at frequency $\mathrm{F}(0.295)$ and a less prominent peak at frequency $\mathrm{F}(0.055)$. The domain 106:130 was next to the right edge of the active site cleft of HA1 and overlapping with epitopes A and B (Table 1), the domain 150:174 contained two binding sites 150 and 152 and overlapping with epitopes $\mathrm{B}$ and $\mathrm{D}$, and the domain 191:221 contained the 190 helix and was overlapping with epitopes B and D. The important locations of these three domains found in HA1 exhibited their significant roles in the binding affinity for 2009 H1N1. As seen from the entropy distribution in Figure 5, these three domains were highly conserved. They were displayed with four different views in the H1 3D model in Figure 3. In [12], a similar domain was found in the
C-terminus of the HA protein consisting of residues 284 -326 .

\subsection{Conserved Domains in HA1 of Swine H1N2}

In [12], the sequences of swine $\mathrm{H} 1 \mathrm{~N} 1$ and $\mathrm{H} 1 \mathrm{~N} 2$ were combined into a single dataset for analysis. Here the swine H1N2 was discussed as a single dataset. We found one domain in HA1 of swine H1N2, which was located in the N-terminus of the protein at residues 1 to 29, and its consensus sequence was DTLCIGYHANNSTDTVDTVLEKNVTVTHS. The domain 1:29 found here was presented with four different views in the H1 3D model in Figure 3, along with the three domains discovered in HA1 in 2009 H1N1. The ISs of the domain 1:29 and the consensus HA1 sequence in swine H1N2 were displayed in Figure 6. The IS of the consensus HA1 sequence in swine H1N2 revealed two dominant peaks at frequencies of $F(0.055)$ and $F(0.295)$. The entropy in Figure 5 suggested that the domain 1:29 was well conserved.

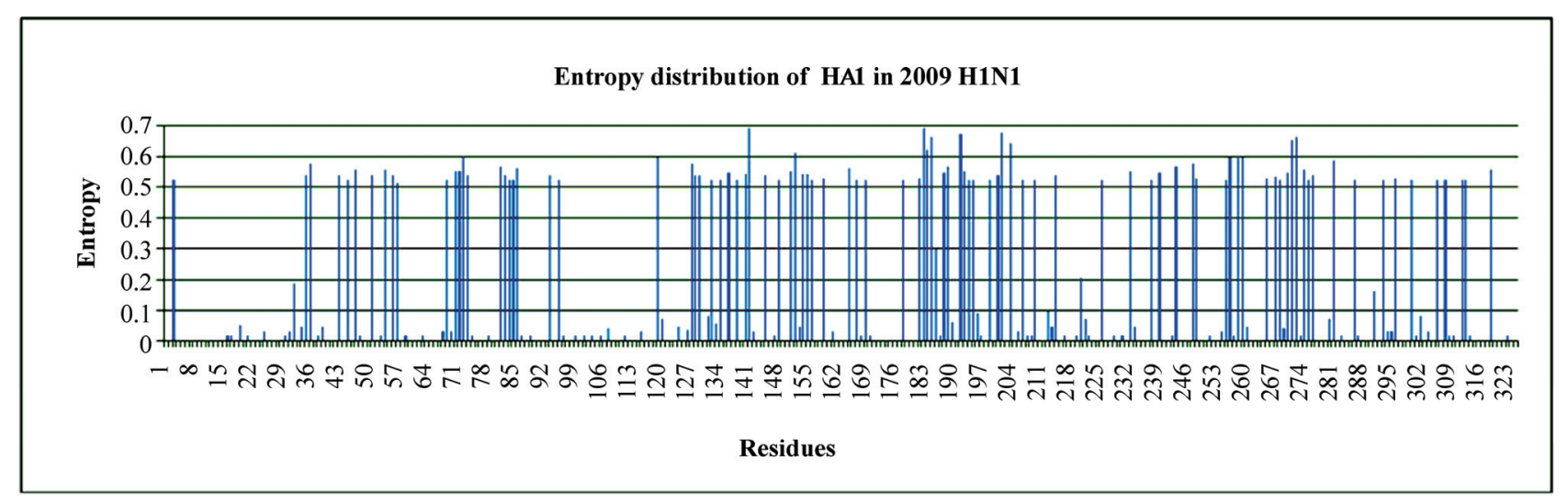

Figure 5. Entropy distribution of HA1 in 2009 H1N1.
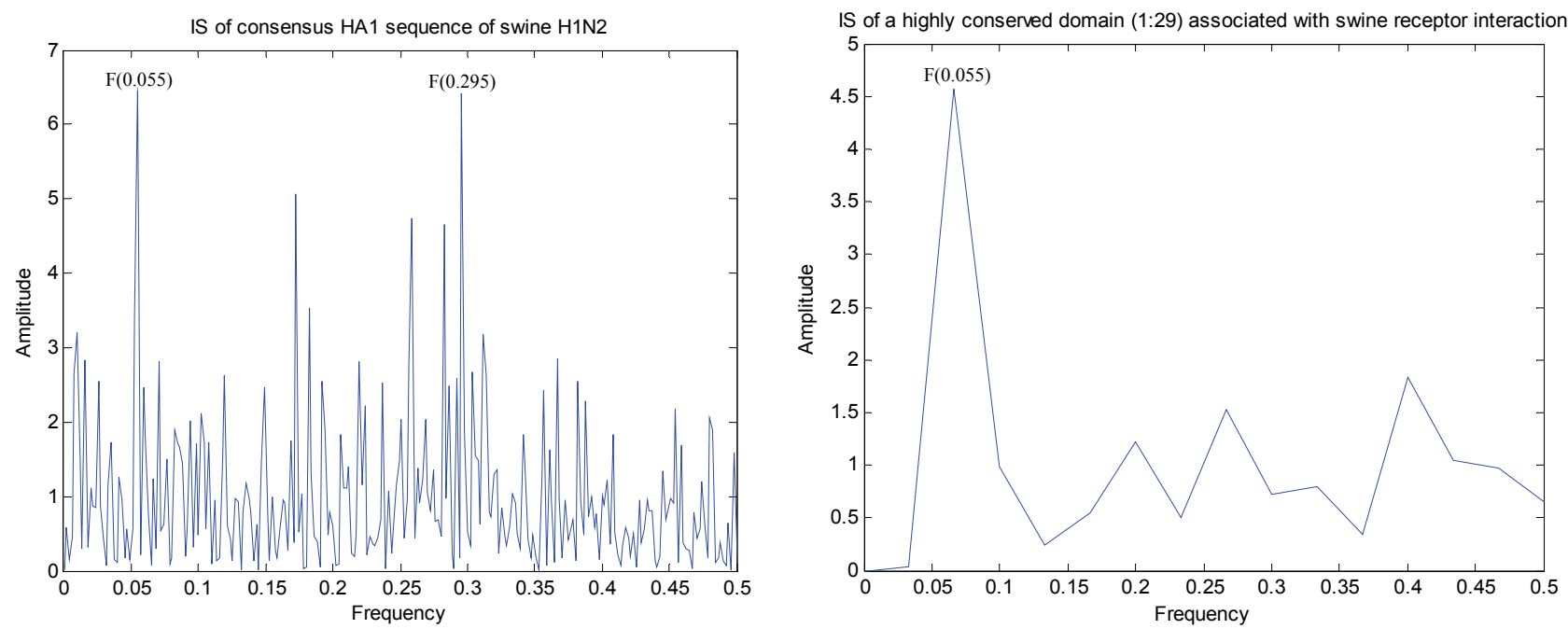

Figure 6. The left plot shows the informational spectrum of consensus HA1 sequence of swine H1N2, and the right plot shows the informational spectrum of one conserved domain 1:29 in swine H1N2 found in this study. 


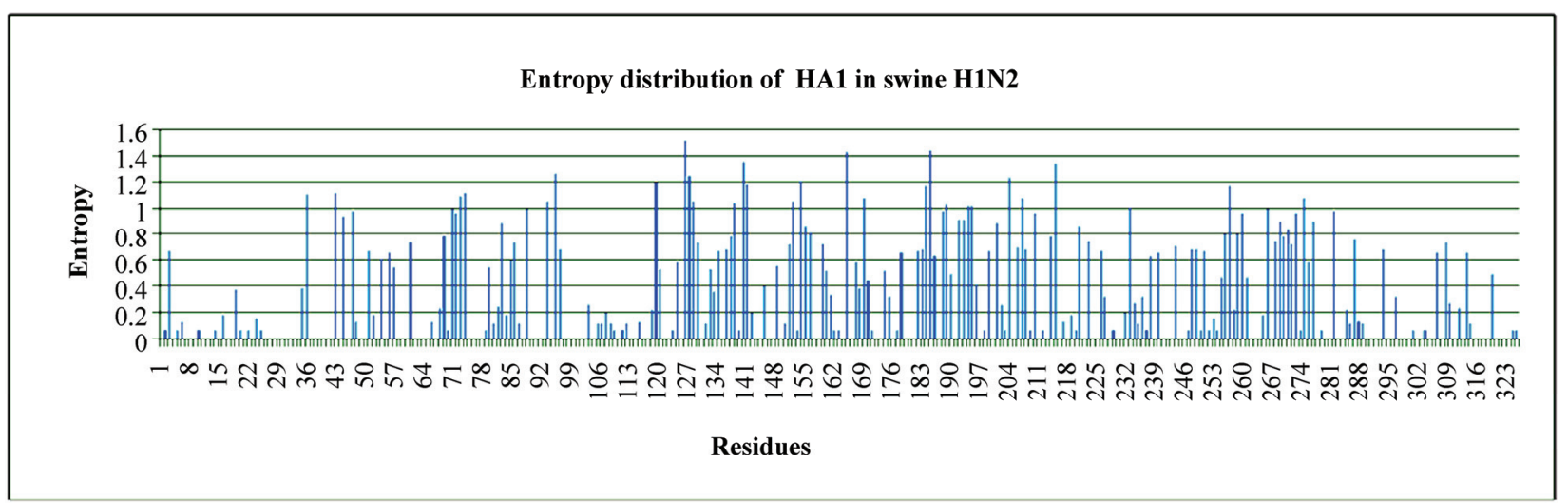

Figure 7. Entropy distribution of HA1 in swine H1N2.
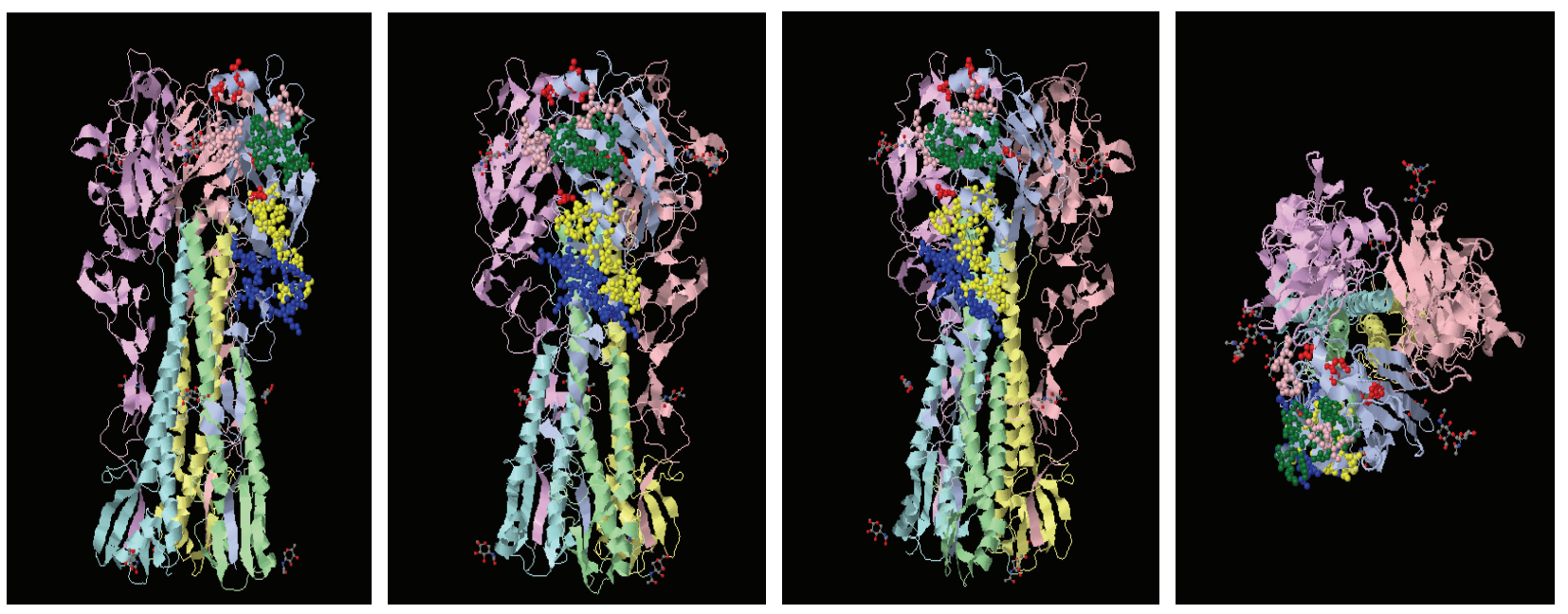

Figure 8. The four plots show in 3D structure the three highly conserved domains found in this study in avian H5N1. Binding sites are colored in red, two edges of the binding cleft in pink, domain 45:65 in yellow, domain 136:153 in green, and domain 269:286 in blue (PDB code: 2IBX).

\subsection{Conserved Domains in HA1 of Avian H5N1}

We uncovered three domains in HA1 of avian H5N1, which were located at residue positions 46 to 65,136 to 153, and 269 to 286. Their consensus sequences were GVKPLILRDCSVAGWLLGNP, PYQGRSSFFRNVVWLIKK, and LEYGNCNTKCQTPMGAIN, respectively, and the ISs of these three domains and the consensus $\mathrm{H}$ A1 sequence in avian H5N1 were illustrated in Figure 9. The IS of the consensus HA1 sequence in avian H5N1 demonstrated two dominant peaks at frequencies $\mathrm{F}$ (0.076) and $F(0.236)$. The three highly conserved domains identified here were exhibited with four different views in the H5 3D model in Figure 8. The entropy distribution in Figure 10 implied that the three domains were well conserved, and the HA1 sequences of avian H5N1 were quite stable, in contrast to that of 2009 $\mathrm{H} 1 \mathrm{~N} 1$ and swine H1N2. In [12], a similar domain was found in the N-terminus of the HA protein comprising residues $42-75$, which encompassed the domain 46:65 found in this study. The epitope mapping analysis in $[19,20,21]$ reported that several anti-HA monoclonal antibodies (MAbs) could recognize the amino acids of $\mathrm{HA}$ at positions $1-86,20-312,136-141,151-162$, and $273-342$, illustrating that our domains might be recognized by these MAbs.

\section{DISCUSSION}

The main findings of this study were presented in Table 5 , which showed the locations, the characteristic frequencies, and the consensus sequences of the highly conserved domains in HA1 discovered in each subtype. The domains in Table $\mathbf{5}$ are shorter than those discovered in $[12,13]$, implying that they were more easily conserved by the HA sequences than their longer counterparts. Furthermore, the identified multiple domains in HA1 could provide more options than those found in the previous studies to design new therapeutic targets for drug development. Finally, because a consensus sequence of each subtype was employed to find these multiple 

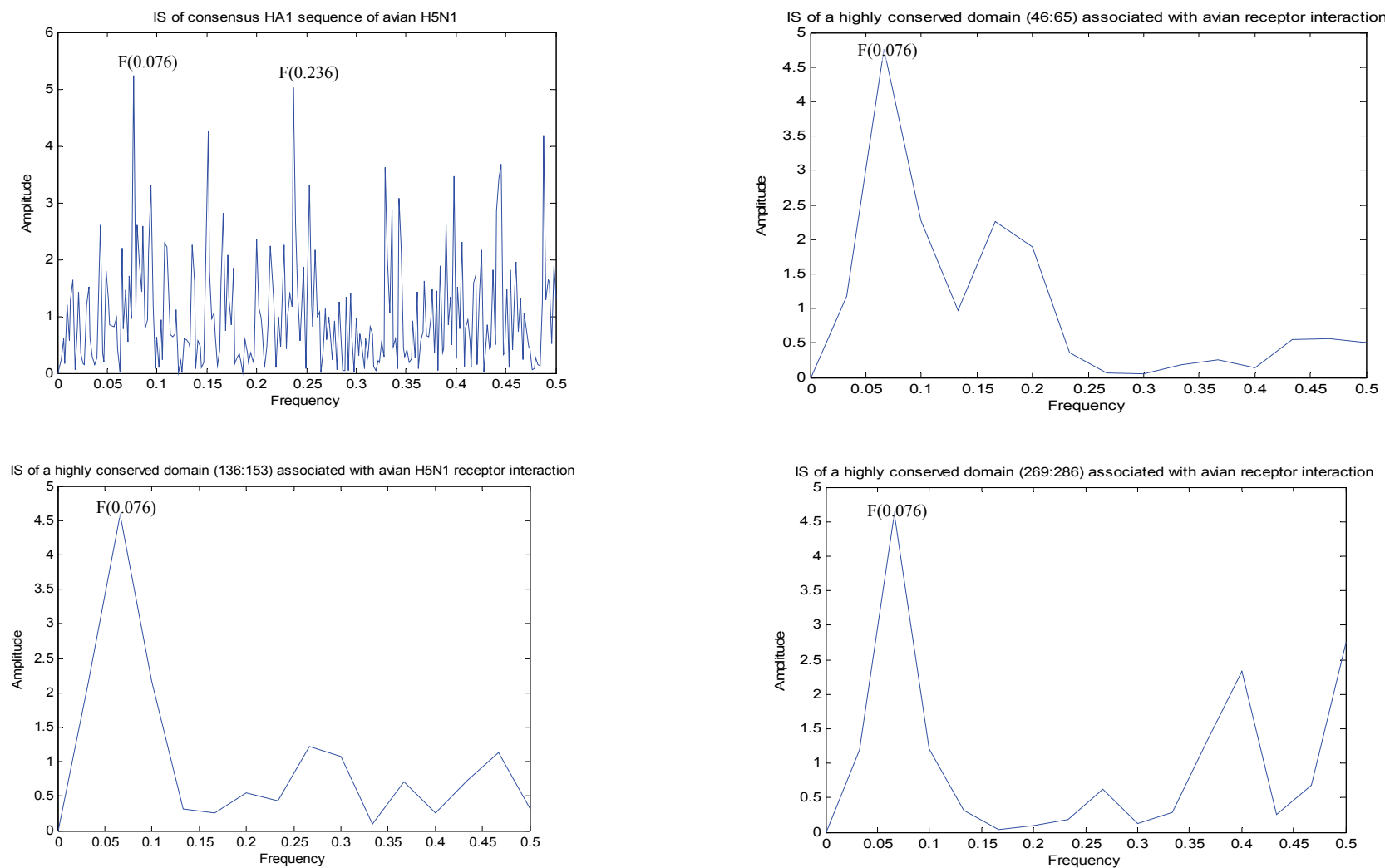

Figure 9. The top left plot shows the informational spectrum of consensus HA1 sequence in avian H5N1. The other three plots show the informational spectrum of the three conserved domains in avian H5N1 found in this study, 46:65, 136:153, and 269:286, respectively.

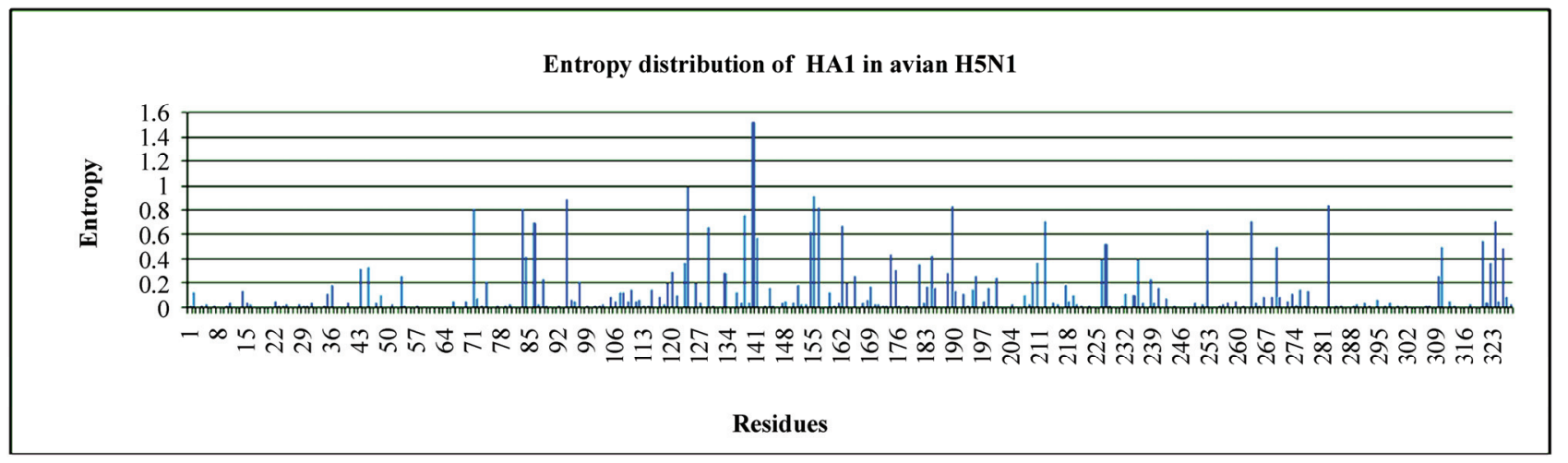

Figure 10. Entropy distribution of HA1 in avian H5N1.

Table 5. The receptor recognition domains of HA proteins in 2009 H1N1, swine H1N2, and avian H5N1 influenza viruses.

\begin{tabular}{cccl}
\hline Subtype & Frequency & Residues & \multicolumn{1}{c}{ Consensus Sequence } \\
\hline 2009 H1N1 & $\mathrm{F}(0.295)$ & $106-130$ & SSVSSFERFEIFPKTSSWPNHDSNK \\
2009 H1N1 & $\mathrm{F}(0.295)$ & $150-174$ & WLVKKGNSYPKLSKSYINDKGKEVLV \\
2009 H1N1 & $\mathrm{F}(0.295)$ & $191-221$ & LYQNADAYVFVGSSRYSKKFKPEIAIRPKVR \\
Swine H1N2 & $\mathrm{F}(0.055)$ & $1-29$ & DTLCIGYHANNSTDTVDTVLEKNVTVTHS \\
Avian H5N1 & $\mathrm{F}(0.076)$ & $46-65$ & GVKPLILRDCSVAGWLLGNP \\
Avian H5N1 & $\mathrm{F}(0.076)$ & $136-153$ & PYQGRSSFFRNVVWLIKK \\
Avian H5N1 & $\mathrm{F}(0.076)$ & $269-286$ & LEYGNCNTKCQTPMGAIN \\
\hline
\end{tabular}


domains in HA1, they are more representative of the strains in each subtype than those obtained in the previous studies.

\section{CONCLUSIONS}

Identifying the conserved characteristics of influenza viruses relevant to receptor binding preference is an important topic in flu research. The informational and structural properties of HA1 associated with receptor-virus interaction in different subtypes were investigated in $[12,13]$. To extend the previous results, we aimed to uncover multiple domains in HA1 of 2009 $\mathrm{H} 1 \mathrm{~N} 1$ and avian $\mathrm{H} 5 \mathrm{~N} 1$ that might modulate the receptor binding patterns, thus to expand our repertoire of these key regions in HA1. Due to the important locations of these domains, they might serve as potential targets for new drugs and treatment of influenza infection.

The observations in $[12,13]$ suggested that the 2009 H1N1 strains will continue to mutate in their HA gene, which could further favor the human interaction pattern by increasing the amplitude at frequency $\mathrm{F}(0.295)$ and at the same time decreasing that at frequency $\mathrm{F}(0.055)$. Given this trend, we must remain vigilant for additional mutations that can render a switch of the binding preference of $2009 \mathrm{H} 1 \mathrm{~N} 1$.

\section{ACKNOWLEDGEMENTS}

We thank Houghton College for its financial support.

\section{REFERENCES}

[1] Alexandra, G., Alexander, T., Galina, P., Nicolai, B., Amanda, B. and Alexander, K. (2006) Evolution of the receptor binding phenotype of influenza A (H5) viruses. Virology, 344, 432-438.

[2] Tatsunori, I., Kaori, F., Katsuhisa, N., Sachiko, A.H., Yuji, M., Hirofumi, W. and Shigenori, T. (2008) Theoretical analysis of binding specificity of influenza viral hemagglutinin to avian and human receptors based on the fragment molecular orbital method. Computational Biology and Chemistry, 32, 198-211.

[3] Shinya, Y., Yasuo, S., Takashi, S., Mai, Q.L., Chairul, A. N.S., Yuko, T., Yukiko, M. et al. (2006) Haemagglutinin mutations responsible for the binding of $\mathrm{H} 5 \mathrm{~N} 1$ influenza A viruses to human-type receptors. Nature, 444(7117), 378-82.

[4] Venkataramanan, S., Kannan, T., Rahul, R., Raguram, S., Zachary, S., Sasisekharan, V. and Ram, S. (2009) Extrapolating from sequence - the $2009 \mathrm{H} 1 \mathrm{N1}$ "swine" influenza virus. Nature Biotechnology, 27, 510-513.

[5] Robert, A.C., Angelina, S.P., Steve, W., Tatyana, M., Liu, Y., Chai, W., Maria, A.C.R., Zhang, Y., Markus, E., Makoto, K., Alan, H., Mikhail, M. and Ten, F. (2009) Receptor-binding specificity of pandemic influenza A (H1N1) 2009 virus determined by carbohydrate microarray. $\mathrm{Na}$ ture Biotechnology, 27, 797-799.

[6] Hu, W. (2009) Analysis of correlated mutations, stalk motifs, and phylogenetic relationship of the 2009 influenza a virus neuraminidase sequences. Journal of Biomedical Science and Engineering, 2(7), 550-558.

[7] Hu, W. (2010) The interaction between the 2009 H1N1 influenza a hemagglutinin and neuraminidase: Mutations, co-mutations, and the NA stalk motifs. Journal of Biomedical Science and Engineering, 3, 1-12.

[8] Cosic, I. (1997) The resonant recognition model of macromolecular bioreactivity - theory and application. Birkhauser Verlag, Berlin.

[9] Veljkovic, V. and Metlas, R. (1988) Identification of nanopeptide from HTLV3, LAV and ARV-2 envelope gp120 determining binding to T4 cell surface protein. Cancer Biochem Biophys, 10, 91-106.

[10] Veljkovic, V., Veljkovic, N., Este, J.A., Huther, A. and ietrich, D.U. (2007) Application of the EIIP/ISM bioinformatics concept in development of new drugs. Curr Med Chem, 14, 441-453.

[11] Veljkovic, V., Veljkovic, N. and Metlas, R. (2004) Molecular makeup of HIV-1 envelope protein. Int Rev Immunol, 23, 383-411.

[12] Veljkovic, V., Niman, H.L., Glisic, S., Veljkovic, N., Perovic, V. and Muller, C.P. (2009) Identification of hemagglutinin structural domain and polymorphisms which may modulate swine H1N1 interactions with human receptor. BMC Structural Biology, 9, 62.

[13] Veljkovic, V., Veljkovic, N., Muller, C.P., Müller, S., Glisic, S., Perovic, V. and Köhler, H. (2009) Characterization of conserved properties of hemagglutinin of H5N1 and human influenza viruses: Possible consequences for therapy and infection control. BMC Struct Biol, 7, 9-21.

[14] Katoh, K., Kuma, K., Toh, H. and Miyata, T. (2005) MAFFT version 5: Improvement in accuracy of multiple sequence alignment. Nucleic Acids Res, 33, 511-518.

[15] MacKay, D. (2003) Information theory, inference, and learning algorithms. Cambridge University Press.

[16] KováccaronOVá, A., Ruttkay-Nedecký, G., HaverlíK, I. K. and Janecccaronek, S. (2002) Sequence similarities and evolutionary relationships of influenza virus a hemagglutinins. Virus Genes, 24, 57-63.

[17] Gamblin, S.J., Haire, L.F., Russell, R.J., Stevens, D.J., Xiao, B., Ha, Y. et al. (2004) The structure and receptor binding properties of the 1918 influenza hemagglutinin. Science, 303, 1838-1842.

[18] Michael, W.D. and Pan, K. (2009) The epitope regions of H1-subtype influenza A, with application to vaccine efficacy. Protein Engineering, Design \& Selection, 22, 543546.

[19] Du, A., Daidoji, T., Koma, T., Ibrahim, M.S., Nakamura, S., de Silva, U.C., Ueda, M., Yang, C.S., Yasunaga, T., Ikutu, K. and Nakaya, T. (2009) Detection of circulating Asian $\mathrm{H} 5 \mathrm{~N} 1$ viruses by a newly established monoclonal antibody. Biochem Biophys Res Commun, 378, 197-202.

[20] Kaverin, N.V., Rudneva, I.A., Govorkova, E.A., Timofeeva, T.A., Shilov, A.A., Kochergin-Nikitsky, K.S., Krylov, P.S. and Webster, R.G. (2007) Epitope mapping of the hemagglutinin molecule of a highly pathogenic $\mathrm{H} 5 \mathrm{~N} 1$ influenza virus by using monoclonal antibodies. J. Virol., 81, 12911-12917.

[21] Yang, M., Clavijo, A., Graham, J., Salo, T., Hole, K., Berhane, Y. (2009) Production and diagnostic application of monoclonal antibodies against influenza virus $\mathrm{H} 5, \mathrm{~J}$ Virol Methods, 162, 194-202. 